\title{
A Covalent Virus Layer For \\ Mass-Based Biosensing
}

\author{
Li-Mei C. Yang, Juan E. Diaz, Theresa M. McIntire, \\ Gregory A. Weiss* and Reginald M. Penner* \\ Department of Chemistry, University of California, \\ Irvine, CA 926970-2025
}

\section{Supplementary Material}

\section{Additional Experimental Methods}

Phage ELISA for determining K07-pAb apparent binding affinity. A Maxisorp immunoplate (96-well) was coated with M13 K07 phage for one hour at room temperature $(100 \mu \mathrm{l}, 10 \mathrm{mM}$ in $50 \mathrm{mM}$ sodium carbonate buffer, $\mathrm{pH}$ 9.6). The plate was then blocked for $30 \mathrm{~min}$ at room temperature with BSA $(0.2 \%)$ in PBS and washed five times with PT buffer (PBS, $0.05 \%$ Tween 20$)$. The coated wells were incubated with anti-M13 monoclonal antibody $(100 \mu \mathrm{l})$ at concentrations ranging from $5 \mathrm{nM}$ to $2 \mathrm{pM}$ in PBS, BSA $(0.1 \%)$, Tween $20(0.1 \%)$ for one hour at room temperature, and washed five times with PT buffer. After washing, the plate was incubated with anti-Mouse IgG/horseradish peroxidase conjugate (100 $\mu \mathrm{l}, 1: 2000)$ in PBS, BSA (0.1\%), Tween 20 $(0.1 \%)$ for one hour, then washed five times with PT and twice with PBS. The plate was developed by using an o-phenylenediamine dihydrochloride/ $\mathrm{H}_{2} \mathrm{O}_{2}$ solution $(100 \mu \mathrm{l}, 1 \mathrm{mg}$ $\mathrm{ml}^{-1} / 0.02 \%$ ), and read spectrophotometrically at $450 \mathrm{~nm}$ (Figure S1).

Mass spectrometric determination of p-Ab molecular weight. A $10 \mu \mathrm{l}$ aliquot of $p$ $A b$ was treated with $50 \mathrm{mM} \mathrm{DTT}$, and incubated for one hour at room temperature. After addition of TFA to $1 \%(\mathrm{v} / \mathrm{v})$, the solution was desalted and concentrated by an Omix C4 pipette tip (Varian) according to the manufacturer's directions. The sample was eluted with addition of $50 \%$ acetonitrile, $49 \%$ water, $1 \%$ TFA saturated with sinnapinic acid before MALDI MS, using a PE Biosystems Voyager system 4124. Measurements from seven samples were averaged together to determine a molecular weight of $148,201 \pm 319 \mathrm{~g} / \mathrm{mol}$ (Figure S2). 


\section{Supplementary Figures}

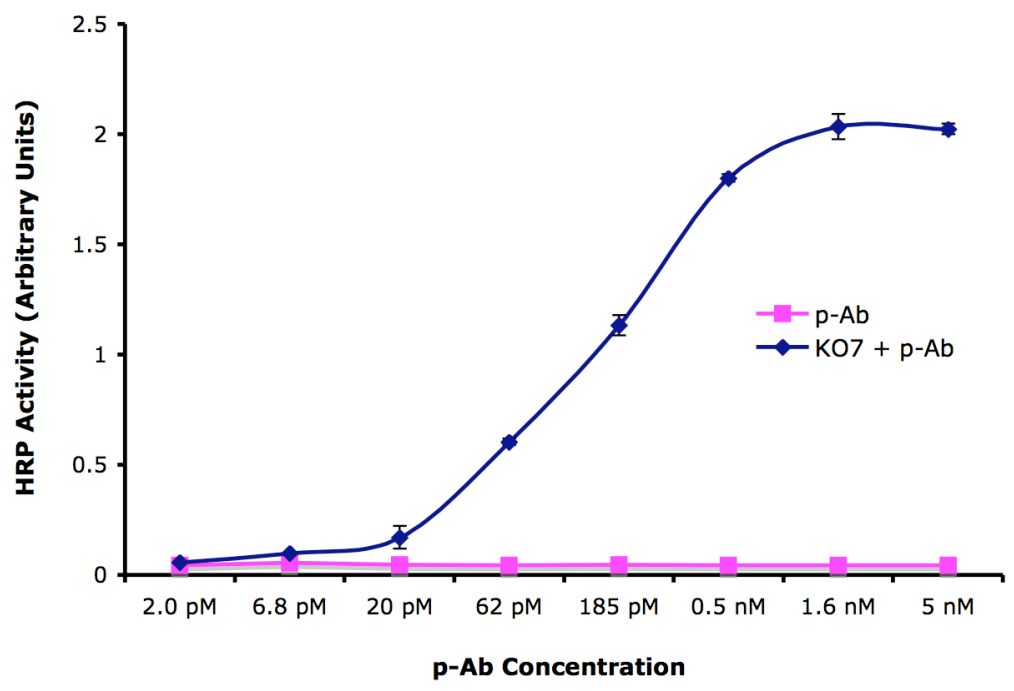

Figure S1. ELISA measurement of the apparent $K_{\mathrm{D}}$ of $\mathrm{p}-\mathrm{Ab}$ binding to $\mathrm{M} 13-\mathrm{K} 07$ phage. Serial dilutions of $\mathrm{p}-\mathrm{Ab}$ were incubated in wells containing immobilized $\mathrm{K} 07$ phage (blue). Negative control wells contained no K07 phage (pink). Anti-mouse IgG antibody conjugated to HRP was used for quantification. Error bars depict the standard error for the average of three independent assays.

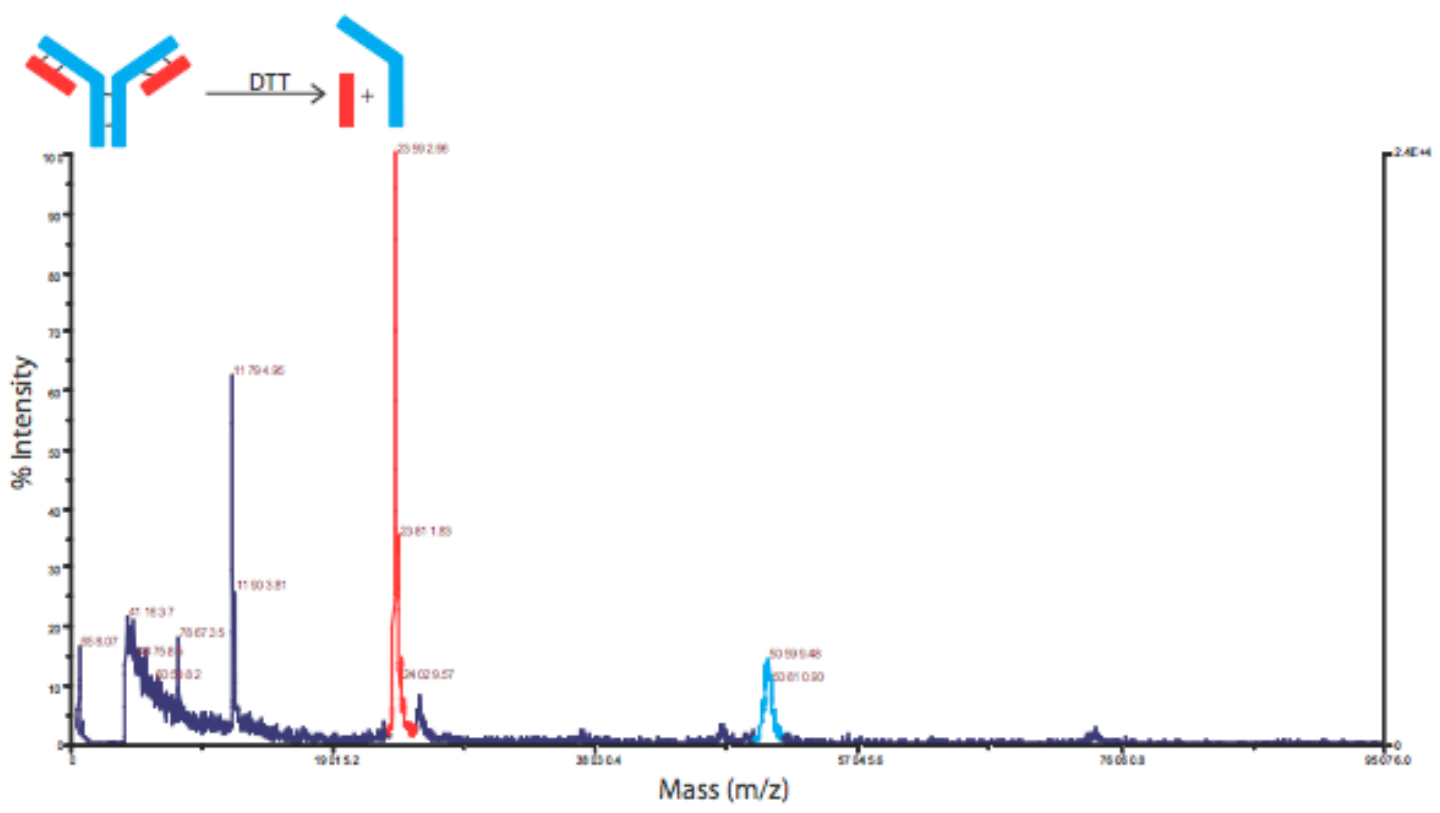

Figure S2. MALDI MS determination of the $\mathrm{p}-\mathrm{Ab}$ molecular weight. After reduction of disulfide bonds with DTT (upper), the masses of p-Ab light (red) and heavy (light blue) chains could be determined. 


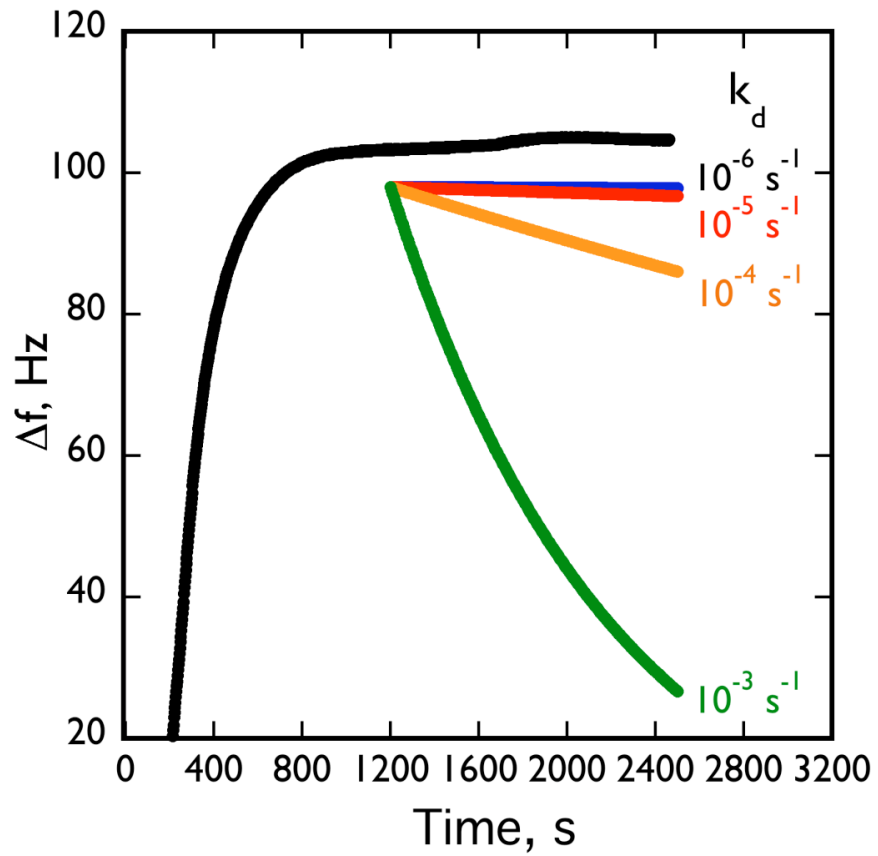

Figure S3. Plot of the $\Delta \mathrm{f}$ versus time for an exposure to $200 \mathrm{nM} \mathrm{p}-\mathrm{Ab}$ showing no perceptible decay in mass (above noise) for 2000 seconds. In color are shown the predicted $\Delta \mathrm{f}$ versus time decays that would be seen for four values of $k_{d}$, ranging from $10^{-6} \mathrm{~s}^{-1}$ to $10^{-3} \mathrm{~s}^{-1}$. The observed mass stability is consistent with a $\mathrm{k}_{\mathrm{d}}$ of $10^{-5} \mathrm{~s}^{-1}$ or less. 